A structured narrative literature review of Approved Mental Health Professional detention decisions: an infusion of morality.

Matthew Simpson

Department of Social Sciences and Social Work, Bournemouth University, Bournemouth, UK.

MSimpson@bournemouth.ac.uk

Matthew Simpson is studying for a PhD at Bournemouth University and working as an AMHP Lead for a local authority in England.

Word count: 6389 


\title{
A structured narrative literature review of Approved Mental Health Professional detention decisions: an infusion of morality.
}

\author{
In England and Wales, Approved Mental Health Professionals (AMHPs) may make \\ applications to detain people in hospital under the Mental Health Act (MHA) 1983 as \\ amended 2007. This paper seeks to establish what the available academic literature has \\ to say about AMHP detention decisions. A structured narrative literature review was \\ conducted with the identification of twenty-five included texts, that were thematically \\ analysed interpretively. Risk, accountability, and morality are identified as dominant \\ themes in this review. Risk is prominent, but on closer inspection risk is infused with \\ morality. Morality is the merging of the personal and professional domains, the use of \\ self to understand another. Accountability is situated between morality and risk, \\ inextricably linked on both sides with authors identifying a fear of responsibility for \\ adverse consequences. Sub-themes of emotions, intuition, uncertainty, coercion, and \\ alternatives (to admission to hospital) were also found. Analysis of nearly thirty years \\ of literature regarding ASW/AMHP detention decisions tells us that they are not based \\ solely on technical judgements; morality permeates the decision. The literature is \\ justified in evincing risk in detention decisions, but the prominence of risk \\ overshadows accountability and belies the influence of morality.
}

Keywords: AMHP; ASW; decision-making; risk; accountability; morality.

\section{Background}

The role of the Approved Social Worker (ASW) was introduced in the England and Wales Mental Health Act (MHA) 1983, the purpose being to bring the social perspective to bear upon detention decisions as advocated for most notably by Gostin (1975) and the British Association of Social Workers (1977). When the MHA 1983 was amended in 2007 the ASW was renamed the Approved Mental Health Professional (AMHP), a role extended beyond social workers to include mental health or learning disability nurses, occupational therapists and psychologists. Its remit did not however change. Not including emergency or criminal justice provisions, detention decisions involve three professionals: a doctor with previous acquaintance if possible; a doctor approved under s 12(2) MHA 1983 as amended 2007 with specialist knowledge in treating mental disorder, two s 12(2) approved doctors if the former 
doctor with previous acquaintance cannot be achieved; and an AMHP. All three have a statutory responsibility in the assessment process: medical recommendations are founded on grounds set out in s 2(2) and s 3(2) Mental Health Act (MHA) 1983 as amended 2007 which include implicit risk criteria including health or safety of the person or the protection of harm to others, which from the analysis has translated into risk being identified as a theme in this review; while the application for detention made by an AMHP is founded upon broader terminology set out in s 13(2) MHA 1983 as amended 2007, including "all the circumstances of the case" which could encompass any relevant factors. The AMHP provides an independent decision (Department of Health 2015, Chapter 14.52) which from the analysis in this review has given rise to accountability being identified as a theme. Both found themes are infused with morality.

A national survey identified ninety-four percent of AMHPs are social workers (Skills for Care 2019). One commentator views social work as moral engagement, emphasising the way social workers connect with other people, "what matters is not only what we know, but what we are" (Whan 1986, 246). His paper argues social work is more than technical knowledge, with the essence of the helping relationship being moral engagement and the moral relationship (Whan 1986, 250). This reaches beyond professional values, merging with the personal domain. Starting with Aristotelian phronesis, which he describes as being concerned with judgements about what is good (Whan 1986, 245), Whan argues that to determine what is good for another we must reach beyond ourselves to understand the other person, representing the enactment of moral knowledge (Whan 1986, 249). Chu et al. (2009, 289) highlight the intersubjective nature of such moral decisions, created between the social worker and service user, and motivated by the commitment to social justice that characterises the social work profession. Another author of social work practice constructs risk as a moral issue (Stanford 2011, 1527). In her study she identified how risk became infused with the 
social worker's personal identity (Stanford 2011, 1525), with moral dilemmas being resolved on a personal level (Stanford 2011, 1525). Morality in this current review is defined in this light, with the literature supporting the notion that AMHPs from all eligible professions studied merge their personal and professional domains, using themselves to build an understanding of service users when making detention decisions.

This structured narrative literature review addresses the search question: what is known about the factors that influence AMHP detention decisions under the MHA 1983 as amended 2007? It will begin with an explanation of the search strategy and rationale followed by an account of the type of review conducted and a discussion of the found themes.

\section{Methods}

\section{Search Strategy}

A start date of 1983 has been applied to reflect the introduction of the ASW role, and an end date of $22^{\text {nd }}$ January 2020 to coincide with the time of this analysis. Papers relating to roles like the AMHP in other jurisdictions with different mental health laws may have been a valuable source for this review topic, however they have been excluded because there are differences in these legal frameworks with unknown influences on decision-making.

An EBSCOhost database search was conducted using Boolean operators. Only peer reviewed academic papers were included to enhance the possibility that the sources would be trustworthy having received validation by experts in the field. The following terms were used: "Approved mental health pr*” OR “approved social work*” AND TI/AB "decision making” OR decision-making OR deciding OR decision\# OR uncertainty OR risk OR experience\# OR complexit*.

This search returned eighty results which reduced to seventy once the duplicates were removed. Wallace and Wray's (2011) critical reading tool was adopted, with a critical 
synopsis undertaken for each apparently relevant text to help scrutinise their quality and relevance, leaving fifteen papers for review.

A search of the British Library e-theses online (EThOS) for additional United Kingdom doctoral theses was conducted using the term 'mental health act assessment'. This returned ninety-two theses, one of which was relevant for inclusion (Skinner 2006).

Reference harvesting identified a further five papers and two books (Sheppard 1990; Peay 2003) which although not peer reviewed were cited many times by other authors strengthening their credibility. A key author search returned another one paper and one thesis resulting in a total of twenty-five texts (so named hereafter due to the inclusion of papers, theses and books) for inclusion.

The literature relating to AMHP decision-making is diverse; many texts include aspects of decision-making that are peripheral to the focus of the paper, and where decisionmaking is discussed the context differs making comparison between these texts more interpretive. Applicable texts include published empirical studies (10), empirical studies as doctoral theses (5), empirical studies published as books (2), and theory papers (8). The gains achieved by increased content for analysis may be limited by the lack of empirical foundation in some of the included texts.

\section{Type of review}

A narrative literature review was selected since it captures the relevant data woven into a complex body of literature (Collins and Fauser 2004). Systematic methods were adopted to provide transparency to the search methods in order to make the review reproducible (Collins and Fauser 2004). Given the inclusion of theory papers Paré et al. $(2015,191)$ suggest the review is structured rather than systematic, enhancing the quality of the review. Whilst the author's experience as a practitioner in the field may have influenced the selection of included texts, the structured methods adopted and transparency in the selection process 
redressed any possible bias. Additionally, Wallace and Wray's (2011) tool for determining the suitability of a text for inclusion in the review was adopted including a critical synopsis of each possible text to develop a clear judgement about whether a text had sufficient relevance to the review question, with a more detailed analysis completed for the remaining key texts once this judgement had been made. What follows is a discussion of the themes arising from this detailed analysis.

\section{Thematic analysis}

All references to factors that influence AMHP detention decisions were coded from the data based on the critical analyses completed for each text (Wallace and Wray 2011). Codes were then grouped to reflect patterned responses and overarching themes were developed to capture the essence of these codes (Braun and Clarke 2006). Thematic analysis was used to seek patterns in these data (Braun and Clarke 2006), a necessarily interpretive process that is likely to have been influenced by the author's experience as an AMHP. Risk, accountability, and morality were identified as dominant themes, with morality infusing all themes. Subthemes of emotions, intuition, uncertainty, coercion, and alternatives were also identified.

\section{Dominant themes}

\section{Risk}

Sheppard considered the implicit risk criteria for detention to be central to the ASW role (Sheppard 1990, preface). His research spanned twelve-months and 120 referrals for assessment. He conducted semi-structured interviews with nine ASWs, adopting a risk analysis methodology. His focus was on the assessment of risk by ASWs, rather than risk arising from his research, as a theme. He highlighted the interpretive nature of risk decisions, subject to variation between ASWs, and he focussed on the notion of a threshold of risk, beyond which detention may be justified (Sheppard 1990). The subjective nature of risk hints toward the personal domain because not all people from the same professional group with the same training made the same decisions. This was also central for Peay, who found variable 
levels of tolerance of risk between participants (Peay 2003, 19). She examined how forty psychiatrists and forty ASWs made detention decisions together, adopting a vignette methodology using a hypothetical case video and notes. Many of her conclusions are generalised across the participants, her theoretical stance is critical toward the legislation, and her use of a hypothetical scenario may have impacted the results albeit she offers a reasonable defence against these potential limitations. Peay (2003) also found ASWs generally wanted to get to know the person more fully prior to deciding about detention, despite being provided with a lot of written information about the person, suggesting a desire for a more personal connection and therefore introducing a moral dimension.

Similarly, Quirk et al. $(2003,125)$ found a greater knowledge of the person being assessed raised the tolerance of risk by ASWs. This observation extended beyond mere information to a more holistic understanding of the individual based on a relationship with them. They carried out a participant-observation fieldwork study spanning fourteen months with twenty assessments being observed in five teams across two London boroughs, one inner-London and one outer-London. Data were analysed using a grounded theory approach. The study was observational and therefore captured ASW practice first-hand. Together the findings from these studies begin to suggest risk does not stand alone, but that AMHP's morally engage with the person being assessed to form situated risk constructions.

Stone $(2017,88)$ identified that lack of knowledge of the person being assessed lowered the risk threshold. He also undertook a vignette study adopting the same case video used by Peay (2003), conducting semi-structured interviews with ten social work and ten nurse AMHPs once they had viewed the video. Understanding of risk assessment was the premise of Stone's study rather than risk arising from the data. Results may have been affected by his sample given the AMHP role still comprises ninety-four percent social workers despite being opened to other, non-medical professionals (Skills for Care 2019), 
however his intention was to compare professional groups. Stone's participants talked of decisions being based more on feelings than information (Stone 2017, 87), indicating a personal connection. Stone additionally found risks were interpreted subjectively by AMHPs (Stone 2017, 140). Glover-Thomas (2011) also identified the significance of knowledge of the person being assessed. She sought to establish whether the legal framework amplified risk, and she adopted an affirmative bias which is supported by her research findings and references to wider literature. She interviewed AMHPs, psychiatrists and former clinical administrative staff then making policy decisions. She does not give detailed participant information or sample size, nor does she discuss her methodology. She identified that participants had a lower risk threshold for detaining people unknown to the service (GloverThomas 2011, 602). All these studies, using a range of methodological approaches but are biased by focus, theoretical stance or methodological vagaries, nevertheless they provide a consistent finding for the value of knowing the person when assessing risk in the context of detention decisions, one aspect of moral engagement where an understanding of risk may emerge through a personal connection.

Yet, Glover-Thomas also identified clinical history as prejudicial (Glover-Thomas 2011, 598), supported later by Abbott $(2018,97)$. Abbott carried out semi-structured interviews of eleven social work AMHPs, therefore excluding other professional groups, providing rich descriptions of case stories which he analysed through framework analysis. All included case stories related to assessments that resulted in detention, which may have given rise to a bias in the results. However, the seeming contradiction regarding the impact of knowing clinical history could be explained by the role of morality in these decisions, where risks may be constructed on a personal level and are therefore subject to more than technical judgements. 
Sheppard's study identified that many ASWs focussed on the patient's mental health, conflating mental illness with risk (Sheppard 1990, 69). This propensity to use a medical approach repeats: O’Hare et al. identified the use of medical dialogues (O'Hare et al. 2013, 201); here case vignettes were used alongside a postal questionnaire which was thematically analysed. Just three ASWs from England and Wales participated in this study (others hailed from different legislative contexts, were student social workers, or AMHP or equivalent trainees), and their conclusions are generalised thereby reducing the applicability of this study to the current review question. Buckland found her participants located treatment in hospitals (Buckland 2016, 54). She carried out semi-structured interviews of ten AMHPs focussing on a recent memorable assessment for them using Foucauldian discourse analysis, a methodology biased toward power imbalance. She found medical narratives being utilised by AMHPs to justify detention (Buckland 2016, 54).

Peay found the contrary; that ASWs spoke more from a social rather than medical perspective (Peay 2003, 196), whilst Hall found that ASWs understood crises in social terms (Hall 2017, 541). In 2008 Hall interviewed service users, ASWs and home treatment professionals involved in nine MHA assessments using framework analysis. This doctoral study was unavailable, but Hall later published from it in 2017. Hall's study was biased toward assessments resulting in home treatment rather than detention, which may have impacted results. He also found ASWs used socially oriented language (Hall 2017, 452) thereby contradicting Sheppard (1990), O’Hare et al. (2013), and Buckland (2016), yet consistent with Peay (2003), suggesting methodological influences, or individual or local operational differences. With some studies highlighting the prominence of social perspectives and some highlighting the prominence of medical perspectives this suggests influences beyond the application of professional values, with personal values being one alternative explanation for the difference. 
The implication from Sheppard (1990), O'Hare at al. (2013) and Buckland (2016) is that a medical orientation is more likely to lead to detention than a social orientation, however Abbott found this was equally true of a social orientation (Abbott 2018, 5).

Sheppard identified a lack of clarity between what is understood by hazards, dangers and risks (Sheppard 1990, 59). It was this lack of clarity that affected the threshold of risk, which he concluded led to variable outcomes (Sheppard 1990). Under similar circumstances some ASWs felt the risk threshold for detention was met where others did not, a finding echoed by Glover-Thomas (2011) who noted that participants could not define risk but viewed it as self-evident (Glover-Thomas 2011, 603), while O'Hare et al. felt participants had an "arbitrary understanding of risk" (O’Hare et al. 2013, 200).

Sheppard developed a decision tool to attempt to address subjectivity and poor clarity, which he later tested among five ASWs undertaking over seventy-one assessments, adopting an action research methodology. He found that consistency and clarity were both improved from using the tool (Sheppard 1993). Glover-Thomas also sought some way of standardising risk assessment; however, she found her participants wanted professional discretion over risk (Glover-Thomas 2011, 597). That decision tools have not become widely used by AMHPs making detention decisions arguably supports the interpretive nature of detention decisions.

Closely aligned to risk in the analysis of the literature in this review is accountability, where the focus of risk concerns the consequences for the individual AMHP given the independence of their decision (Department of Health 2015, Chapter 14.52).

\section{Accountability}

Skinner $(2006,98)$ found that professional liability was a significant factor in detention decisions. She sought to understand the experience of ASWs, senior nurse practitioners, and psychiatrists carrying out MHA assessments, using grounded theory. She interviewed eleven participants from one geographical region, seven of whom were ASWs. 
Quirk et al. found that peer support reduces the likelihood of detention (Quirk et al. 2003, 125). Gregor found ASWs experienced stress from their responsibility in being required to make detention decisions (Gregor 2010, 439). She interviewed twenty-five ASWs using grounded theory, focussing on how they related emotionally to the role. ASW detention decisions were ancillary to her study, but she found working in a supportive team was valued (Gregor 2010, 440).

Kinney explored the ethical issues involved in the detention process, contending that, in the face of two medical recommendations for detention, promoting autonomy is ceded to concerns about accountability for risk-taking (Kinney 2009, 332). Written from his experience as a practitioner, Kinney's theoretical paper is biased toward issues of power, oppression and anti-discriminatory practice. He, crucially, identifies that at the same time as being accountable for the risk-taking, AMHPs have little power to influence risk-taking in less controlling ways, leading to ethical compromise (Kinney 2009, 334). Risk and accountability may then present moral conflict where alternatives are unavailable.

O'Hare et al. hypothesised a risk averse climate where future risks are overestimated (O'Hare et al. 2013, 201). Buckland supported this, with all her participants linking public discourses on risk to defensive risk averse practice due to personal accountability (Buckland 2016, 57-8). Quirk et al. identified the impact of the 'blame culture', with a detention decision being favoured to avoid censure (Quirk et al. 2003, 128). Organisational culture may then influence detention decisions through the AMHP's perception of accountability (whether real or imagined).

Fistein et al. identified that practitioners feared accountability for a person's future behaviour (Fistein et al. 2016, 55). They took a mixed-methods approach adopting an institutional ethnography in their observation of discussions between medical practitioners and AMHPs, following assessments. This they combined with individual narrative interviews 
with those practitioners using a biographical narrative interpretive methodology. Their study involved a limited number of AMHP participants: while five AMHPs were involved in the observational component only one AMHP was interviewed individually. Nevertheless, this finding is consistent with wider research. In a later paper Glover-Thomas theorised that a fear of adverse consequences influences detention decisions (Glover-Thomas 2018, 13).

These findings, it is suggested, unite risk, and accountability in detention decisions underpinned by notions of morality and moral conflict.

\section{Morality}

Quirk (2007) includes additional analysis on an earlier study conducted by himself and colleagues (Quirk et al. 2003). He proposed the resources made available to provide alternatives to admission are crucial to the detention decision, making the institutional context of direct relevance (Quirk 2007, 302). Quirk et al. (2003) established that organisational culture operates on a team level, finding local operational norms in some geographic areas that promote detention as a last resort, while in other areas with less resources there was a sense of pragmatism favouring detention (Quirk et al. 2003, 127-128). Arguably, a moral position that supports detention as a last resort contradicts a moral position that views detention as pragmatic, suggesting that organisational culture may influence individual moral positions.

Kinney provided an example of detention in the absence of alternative resources leading to his sense of personal failure (Kinney 2009, 334), a direct acknowledgement of the personal domain. For Kinney the morals of his organisation were at odds with his own, a position far removed from pragmatism. He concludes the main resource in an MHA assessment is the AMHP themselves (Kinney 2009, 336), one aspect of moral engagement.

Dwyer commented that the detention process "can feel like a barbarity" (Dwyer 2012, 350). She wrote a theory paper to illuminate her experience of AMHP practice, with moral 
conflict operating internally rather than clashing with organisational values. Her stark emotional response to detention highlights the decision is made on a personal as well as a professional level.

Quirk (2007) explored the notion of dirty work in relation to the ASW role, where perhaps moral conflict might have been expected if a clash in organisational and individual morals were present. Dirty work was a term coined by Hughes (1971) to refer to morally dubious work. This concept directly bridges the personal and professional domains. While the ASW role as dirty work was acknowledged by participants in the study, a more prominent perspective was one that viewed detention as helpful to people (Quirk 2007, 300).

The notion of dirty work was explored further by Morriss (2016), who conducted narrative interviews with seventeen practicing social work AMHPs analysed using dialogical narrative analysis. She purposefully selected social workers excluding the influence of other AMHP professional groups, possibly affecting her results. She found social work AMHPs recognised the negative impact of admission, particularly that wards can be dangerous (Morriss 2016, 716). The risk of admission was highlighted earlier by Smith $(2001,28)$ who highlighted in his theory paper the risks to self-esteem, harm from treatment and harm from others when in hospital. Kinney $(2009,334)$ similarly highlighted concerns about the risk of admission, both in terms of the environment and the negative impact of treatment and labelling. Morriss links the potential harm caused by admission with the "contradiction inherent in AMHP work" (Morriss 2016, 713), where the negative consequences of detention are balanced against the perceived reduction in harm to the person. Skinner (2006) explored this issue, conceptualising the ethical dilemma as an internal conflict where cognitive dissonance is generated. She found support for the control to care rationalisation to resolve this internal conflict, equally espoused by Yianni (2009) in his theory paper, and Buckland (2016, 52-53). Such a shift suggests morality is pivotal in detention decisions, where AMHPs 
may shift their personal moral position as a way of rationalising their role. Additionally, Buckland identified the importance of personal values which differed between participants (Buckland 2016, 56), a direct reference to a moral dimension.

Fistein et al. (2016) found some support for the rights-based perspective, identifying that participants favoured 'soft paternalism', where decisions were based on the best interests of the person being assessed. For Fistein et al. (2016) moral conflict only occurred with 'hard paternalism', where the risk of harm to others was the principal driver for the detention decision. In his theory paper Campbell highlighted the impossibility of AMHPs balancing the competing expectations of the person, their family, and the public (Campbell 2010, 331-332).

Peay (2003) observed that ASWs became less sure about detention after watching the case video, indicating a greater degree of connection with the individual after seeing them. Dwyer (2012) supported the person-centred nature of AMHP practice, as did Vicary who found support for a positive focus on the person being key to AMHP practice (Vicary 2017, 223). She conducted semi-structured interviews including the drawing of rich pictures with twelve AMHPs, comprising five social workers, five nurses and two occupational therapists. This purposive sample is not representative of the AMHP population given ninety-four percent of AMHPs are social workers (Skills for Care 2019). However, interpretative phenomenological analysis seeks an understanding of a phenomenon, in her case how the role is experienced between professional groups. She identified that positive feelings translated into action, with AMHPs defending the person being assessed from the negative views of others, conceptualising this as a moral concern for others leading to moral action, and identifying this as enacted personhood (Vicary 2017, 223-224).

\section{Sub-themes}

Sub-themes were identified that emerged from the analysis of one or more of the dominant themes. These are: emotions, intuition, uncertainty, coercion, and alternatives. Each sub- 
theme will be discussed and linked to one or more of the dominant themes.

\section{Emotions}

Fatigue was identified by Haynes (1990) as a barrier to thoroughly investigating a situation and a tendency to agree with doctors. Haynes asked participants what influenced their decision in their most recent out of hours assessment. She conducted semi-structured interviews with twelve ASWs, though she has not been explicit about her methodology. Subsequent literature does not mention fatigue as a factor in detention decisions, though Haynes' is the only study in this review to focus on out-of-hours assessments.

Peay (2003) identified the decision to detain is driven by fear of the consequences of not detaining in relation to risk. Fear emerges from risk and accountability in this observation.

Thompson adopts a psychodynamic framework in his theory paper invoking the concept of the ASW containing mental distress (Thompson 2003, 40). Gregor similarly identified the ASW containing the anxieties of others (Gregor 2010, 438). Dwyer supported this concept, adding that decisions are both cognitive and emotional (Dwyer 2012, 346). Morriss developed this further identifying guilt about detention decisions, but that this emotion was how it should be (Morriss 2016, 709). This was echoed by Stone (2017, 118). Guilt is a personal emotional response reaching beyond the professional domain. Vicary introduced what she described as the feeling rule dichotomy whereby, conflicting emotions are used to control practice, so to extend Morriss' example guilt may be used alongside pride to give due weight to a decision (Vicary 2017). Emotions may then operate as an extension to morality, moving beyond professional values and bringing the AMHP as a person closer to their detention decision.

\section{Intuition}

The emotional concept of the 'right decision' arose in some studies (Dwyer 2012; Buckland 
2016; Morriss 2016), with Stone $(2017,118)$ offering the fear of the 'wrong decision' as pervasive. Dwyer (2012) used the term 'emerges' to describe decision-making, which supports a link to intuition. Kinney (2009) referred to suspicions and feelings about what needed to happen, while Glover-Thomas (2011) concluded factors were filtered through intuition to reach a decision. Stone $(2017,117)$ found support for intuition affecting decisionmaking, commenting this was difficult for participants to articulate. Buckland (2016) found participants used the term 'common sense' to describe their decision making, invoking bodily metaphors. The literature supports that the AMHP intuitively uses themselves as a resource in decision-making, by extension integrating intuition with morality.

The related concept of practice wisdom arose in some studies (Dwyer 2012; Vicary 2017; Stone 2017). Peay (2003) may have found some support for the role of intuition in her observation that among ASW's initial impressions were pervasive, and confidence increased with more information.

The concept of identity offers a natural progression from intuition that has been considered by some authors in this review. Skinner (2006) identified the assimilation of the process of MHA assessments into professional identity. Vicary (2017) found the concept of personhood becomes embodied by the AMHP. Stone (2017) commented on the intrinsic nature of the role borne out of "familiarity of undertaking AMHP work" (Stone 2017, 117). The role of intuition and identity highlights how AMHP detention decisions are more than technical in nature, with a blurring of influences that may emerge from the personal as well as the professional domain.

\section{Uncertainty}

Uncertainty is espoused as central to decisions in some of the included texts. Peay inferred risk aversion from uncertainty (Peay 2003, 30). Glover-Thomas (2018) equally provided a perspective that detention will be favoured by professionals where there is uncertainty. These 
studies support a direct link to accountability. Skinner (2006) based her thesis on the premise that uncertainty is central to the assessment process, with Buckland $(2016,46)$ concurring.

\section{Coercion}

The notion of coercion being applied to AMHPs is prevalent within the literature. Haynes (1990) highlighted conflict between GPs and ASWs over detention decisions. In his theory paper Thompson (1997) reflected on a situation in which pressure from his employer impacted his decision-making as an ASW. Thompson (2003) cited an ASW feeling under management pressure to detain someone. Campbell echoed these experiences, surmising that such pressure is likely to compromise decisions (Campbell 2010, 332). Such coercion relates closely to organisational culture discussed in the morality theme, and the link to accountability is implicit. Skinner found her participants reported feeling coerced (Skinner 2006, 113), with Quirk observing that community psychiatric nurses were informally influential over detention decisions (Quirk 2007, 267). Kinney (2009) reflected on his desire for a holistic assessment being delegitimised by others. Abbott highlighted "an adversarial atmosphere at the point of receiving the referral" (Abbott 2018, 148).

\section{Alternatives}

Kinney (2009) conceptualised the absence of power over alternative resources as undermining the AMHP role, a position echoed by Hall (2017) in which he highlighted the ASWs only power is to detain. Hall positioned the ASW as "negotiator and deal-maker in exploring community provision" (Hall 2017, 453). Such a position retains some optimism. However, others cite the limited choice of alternatives to admission as deterministic (Haynes 1990; Quirk at al. 2003; Buckland 2016; Stone 2017; Glover-Thomas 2018). Quirk et al. (2003) problematised a lack of time to organise alternatives, reinforced by Kinney (2009) citing pressure to resolve a situation quickly. 
Thompson (1997) cited the willingness of the service user to work with him as the ASW being decisive in avoiding detention. Hall (2017) evoked the same message, with the ASW as negotiator brokering agreements with the service user, such as to work with the home treatment team. Abbott (2018) added the element of trust to such willingness. Here morality may be the underlying factor through the connection between the AMHP and the service user, constructing situated judgements through moral engagement. Abbott identified the importance of service users accepting they are unwell (Abbott 2018, 128), linking this to trust in them taking medication. Stone (2017) also found medication concordance was an important factor in detention decisions returning to the medical perspectives discussed earlier.

Thompson (1997) reflected that self-control of the person being assessed regarding potential risk behaviour was important for the viability of community options. Abbott (2018) identified risks being invoked to justify detention, and alternatives only being an option if the person being assessed is willing to adhere to the alternative plan. Key to such feasibility arguments was the ability of the family to cope with the person in the community, without which detention was sought (Abbott 2018). Alternatives to detention, then, cohabit risk and accountability and are infused with morality in detention decisions.

\section{Conclusion}

This literature review has addressed the search question: what is known about the factors that influence AMHP detention decisions under the MHA 1983 as amended 2007? A structured narrative literature review was adopted, and twenty-five texts were identified.

Through interpretive thematic analysis risk, accountability, and morality are identified as dominant themes. Risk is a prominent theme echoing the grounds for medical recommendations in s 2(2) and s 3(2) MHA 1983 as amended 2007, but on closer inspection risk is infused with morality, more aligned with the broader grounds for the AMHP making an application in s 13(2) MHA 1983 as amended 2007 "all the circumstances of the case". 
Wide variation is acknowledged; issues such as the social or medical orientation of the AMHP are relevant; and a knowledge of the person is significant. Risk cannot be viewed as an objective matter, rather it is subjective and interpretive, infused with morality. Morality in this review is encapsulated by AMHPs merging their personal and professional domains, using themselves to understand service users when making detention decisions. Between morality and risk lies accountability, inextricably linked on both sides with authors identifying a fear of responsibility for adverse consequences.

Sub-themes of emotions, intuition, uncertainty, coercion, and alternatives are also present. These emerge from one or more of the dominant themes and whilst they have been afforded secondary status in this review, they nonetheless provide further insight into the review question.

Comparing and contrasting nearly thirty years of literature regarding ASW/AMHP detention decisions what is clear from this structured narrative review is that they are not based solely on technical judgements. The literature is justified in evincing risk in detention decisions, but the prominence of risk overshadows accountability and belies the influence of morality. The pervasive nature of morality in AMHP detention decisions is highlighted for the first time in this review, raising the question of whether services are structured in a way that supports AMHPs to achieve moral engagement in their role?

\section{Acknowledgements}

With thanks to Dr Sarah Vicary for her advice and guidance, enabling me to bring greater clarity to this review.

\section{References}

Abbott, Simon. 2018. "Using the Law in Social Work Approved Mental Health Professional 
Practice.” DSW diss., University of Sussex.

British Association of Social Workers (BASW), 1977. Mental health crisis services - A new philosophy. Birmingham: BASW.

Braun, Virginia., and Victoria Clarke. 2006. "Using Thematic Analysis in Psychology." Qualitative Research Psychology 3 (2): 77-101. doi:10.1191/1478088706qp063oa.

Buckland, Rosie. 2016. "The Decision by Approved Mental Health Professionals to Use Compulsory Powers Under the Mental Health Act 1983: A Foucauldian Discourse Analysis.” British Journal of Social Work 46 (1): 46-62. doi:10.1093/bjsw/bcu114.

Campbell, Jim. 2010. "Deciding to Detain: The Use of Compulsory Mental Health Law by UK Social Workers.” British Journal of Social Work 40 (1): 328-334. doi:10.1093/bjsw/bcp121.

Chu, William C. K., Ming-sum Tsui, and Miu-chung Yan. 2009. "Social work as a moral and political practice.” International Social Work 52 (3): 287-298. doi: $10.1177 / 0020872808102064$.

Collins, John. A., and Bart C. J. M. Fauser. 2005. "Balancing the Strengths of Systematic and Narrative Reviews." Human Reproduction Update 11 (2): 103-104. doi:10.1093/humupd/dmh058.

Department of Health, 2015. Mental Health Act 1983: Code of Practice. London: The Stationery Office.

Dwyer, Sandra. 2012. "Walking the Tightrope of a Mental Health Act Assessment." Journal of Social Work Practice 26 (3): 341-353. doi:10.1080/02650533.2011.629293. 
Fistein, Elizabeth C., Isabel C. H. Clare, Marcus Redley, and Anthony J. Holland. 2016. “Tensions Between Policy and Practice: A Qualitative Analysis of Decisions Regarding Compulsory Admission to Psychiatric Hospital.” International Journal of Law and Psychiatry 46: 50-57. doi:10.1016/j.ijlp.2016.02.029.

Glover-Thomas, Nicola. 2011. “The Age of Risk: Risk Perception and Determination Following the Mental Health Act 2007.” Medical Law Review 19 (4): 581-605. doi:10.1093/medlaw/fwr023.

Glover-Thomas, Nicola. 2018. "Decision-Making Behaviour under the Mental Health Act 1983 and Its Impact on Mental Health Tribunals: An English Perspective.” Laws 7 (2) 12: 1-23. doi:10.3390/laws7020012.

Gostin, L., 1975. A Human Condition. Volume 1. London: Mind.

Gregor, Claire. 2010. "Unconscious Aspects of Statutory Mental Health Social Work: Emotional Labour and the Approved Mental Health Professional.” Journal of Social Work Practice 24 (4): 429-443. doi:10.1080/02650531003791012.

Hall, Peter. 2017. "Mental Health Act Assessments - Professional Narratives on Alternatives to Hospital Admission.” Journal of Social Work Practice 31 (4): 445-459. doi:10.1080/02650533.2017.1394825.

Haynes, Ruth. 1990. “After 1983: Approved Social Workers’ Perceptions of Their Changing Role in Emergency Psychiatric Assessments.” Practice 4 (3) 184-198. doi:10.1080/09503159008416894.

Hughes, Everett, C. 1971. The sociological eye: selected papers. Chicago: Aldine-Atherton.

Kinney, Malcolm. 2009. "Being Assessed under the Mental Health Act - Can it Ever Be 
Ethical?” Ethics and Social Welfare 3 (3): 329-336. doi:10.1080/17496530903209584.

Morriss, Lisa. 2016. “AMHP Work: Dirty or Prestigious? Dirty Work Designations and the Approved Mental Health Professional.” British Journal of Social Work 46 (3): 703-718. doi:10.1093/bjsw/bcv009.

O’Hare, Philip, Gavin Davidson, Jim Campbell, and Michael Maas-Lowit. 2013. "Implementing Mental Health Law: A Comparison of Social Work Practice Across Three Jurisdictions." The Journal of Mental Health Training, Education and Practice 8 (4): 196207. doi:10.1108/JMHTEP-12-2012-0044.

Paré, Guy, Marie-Claude Trudel, Mirou Jaana, and Spyros Kitsiou, 2015. "Synthesizing information systems knowledge: A typology of literature reviews.” Information and Management, 52, 2, 183-199. doi: 10.1016/j.im.2014.08.008.

Peay, Jill. 2003. Decisions and Dilemmas: Working with Mental Health Law. Oxford: Hart.

Quirk, Alan. 2007. “Obstacles to Shared Decision-Making in Psychiatric Practice: Findings from Three Observational Studies. PhD diss., Brunel University.

Quirk, Alan, Paul Lelliott, Bernard Audini, and Katie Buston. 2003. "Non-Clinical and ExtraLegal Influences on Decisions about Compulsory Admission to Psychiatric Hospital.” Journal of Mental Health 12 (2): 119-130. doi:10.1080/0963823031000103434.

Sheppard, Michael. 1990. Mental Health: The Role of the Approved Social Worker. Sheffield: Joint Unit for Social Services Research.

Sheppard, Michael. 1993. "Theory for Approved Social Work: The Use of the Compulsory Admissions Assessment Schedule.” British Journal of Social Work 23 (3): 231-257. doi:10.1093/oxfordjournals.bjsw.a055973. 
Skills for Care, 2019. The approved mental health professional workforce in the adult care sector [online]. Leeds: Skills for Care. https://www.skillsforcare.org.uk/adult-social-care$\underline{\text { workforce-data/Workforce-intelligence/documents/AMHPs-Briefing.pdf }}$

Skinner, Laura. 2006. "Negotiating Uncertainty: Mental Health Professionals' Experiences of the Mental Health Act Assessment Process.” DClinPsych diss., University of Leicester.

Smith, Martin. 2001. "Risk assessment in mental health work". Practice, 13:2, 21-30, doi: $10.1080 / 09503150108411508$

Stanford, Sonya., N., 2011. “Constructing Moral Responses to Risk: A Framework for Hopeful Social Work Practice.” British Journal of Social Work 41 (8): 1514-1531. doi:10.1093/bjsw/bcr030.

Stone, Kevin. M. C. 2017. "Decisions on Risk and Mental Health Hospital Admission by Approved Mental Health Professionals. DSocSci diss., University of Bristol.

Stone, Kevin. 2019. “Approved Mental Health Professionals and Detention: An Exploration of Professional Differences and Similarities." Practice 31 (2): 83-96. doi:10.1080/09503153.2018.1445709.

Thompson, Paul. 1997. “Approved Social Work and Psychotherapy.” Practice 9 (2) 35-46. doi:10.1080/09503159708411645.

Thompson, Paul. 2003. "Devils and Deep Blue Seas: The Social Worker In-Between.” Journal of Social Work Practice 17 (1): 35-47. doi:10.1080/0265053032000071466.

Vicary, Sarah. 2017. “An Interpretive Phenomenological Analysis of the Impact of Professional Background on Role Fulfilment: A Study of Approved Mental Health Practice.” PhD diss., University of Manchester. 
Wallace, Mike, and Alison Wray. 2011. Critical Reading and Writing for Postgraduates. $2^{\text {nd }}$ Edition. Thousand Oaks: Sage.

Whan, Michael. 1986. “On the Nature of Practice.” British Journal of Social Work 16 (2): 243-250. doi:10.1093/oxfordjournals.bjsw.a055193.

Yianni, Chris. 2009. “Aces High: My Control Trumps your Care.” Ethics and Social Welfare, 3, 3, 337-343. doi: 10.1080/17496530903209618. 